\title{
Natural biomass as carbon sources for the synthesis of photoluminescent carbon dots
}

\begin{abstract}
Photoluminescent carbon dots (CD), one of the nanomaterial family members with a size of < $10 \mathrm{~nm}$, have become increasingly popular in the past decade due to its unique optical and electric properties. Semiconductor quantum dots that were developed in the earlier years consist of toxic heavy metal elements in its structure, thus rendering it to be applied in environmental and biological application. Due to this drawback, CD acts as promising alternatives to overcome this shortcoming. Over and above, CD presented low toxicity, high biocompatibility, ease of functionalization, and tunable luminescence. Respectively, CD has shown a great potential to be widely applied in various application. In this chapter, a brief summary of CD background was discussed. The use of natural carbon-based materials as a starting material and different approaches on the preparation of $C D$ were summarized. A summary of CD structure and properties was also discussed briefly.
\end{abstract}

Keyword: Carbon dots; Biomass; Biochar; Top-down approach; Bottom-up approach; Photoluminescence 